\title{
Low-risk donor lungs optimize the post-lung transplant outcome for high lung allocation score patients
}

\author{
Takeshi Kurosaki ${ }^{1,2} \cdot$ Kentaroh Miyoshi $^{1} \cdot$ Shinji Otani ${ }^{1,2} \cdot$ Kentaro Imanishi $^{1} \cdot$ Seiichiro Sugimoto ${ }^{1}$. \\ Masaomi Yamane $^{1} \cdot$ Motomu Kobayashi $^{3} \cdot$ Shinichi Toyooka $^{1} \cdot$ Takahiro Oto $^{1,2}$
}

Received: 1 February 2018 / Accepted: 27 April 2018 / Published online: 11 May 2018

(c) Springer Nature Singapore Pte Ltd. 2018

\begin{abstract} influenced by the quality of the donor lungs. LAS and posttransplant outcomes were investigated in both groups. low-risk donor are transplanted.

$\begin{array}{ll}\text { Abbreviations } \\ \text { CDLTx } & \text { Cadaveric lung transplant } \\ \text { DS } & \text { Lung donor score } \\ \text { LAS } & \text { Lung allocation score } \\ \text { LDLLTx } & \text { Living donor lobar lung transplant } \\ \text { LTx } & \text { Lung transplant } \\ \text { OPTN } & \text { Organ Procurement and Transplant Network }\end{array}$
\end{abstract}

Purpose The lung allocation score (LAS) has been generally recognized as a contributor to the overall survival in lung transplant candidates. However, donor-related risks have never been taken into consideration in previous research that validated the LAS. This study aimed to determine whether or not the role of the LAS as a predictor of the posttransplant outcome is

Methods We retrospectively reviewed 108 patients who underwent lung transplantation at Okayama University Hospital since 1998. The cohort was divided into two groups based on the lung donor score (DS; $\leq 4 />4$ ). Correlations between the

Results In the high-DS group, an elevated LAS was strongly associated with posttransplant $\mathrm{PaO}_{2} / \mathrm{FiO}_{2}(p=0.018)$. However, in the low-DS group, no correlation was found between them. There was no significant difference in the long-term survival according to the LAS in the low-DS group. The LAS effectively predicted the posttransplant outcome only when lungs with DS > 4 were transplanted; the LAS was not reliable if high-quality lungs were transplanted.

Conclusion Lung transplantation can be feasible and provides a survival benefit even for high-LAS patients if lungs from a

Keywords Lung transplantation $\cdot$ Lung allocation score $\cdot$ Donor score $\cdot$ Low-risk donor

Kentaroh Miyoshi

kentarohmiyoshi@yahoo.co.jp

1 Department of Thoracic Surgery, Okayama University Hospital, 2-5-1 Shikata-cho, Kita-ku, Okayama 700-8558, Japan

2 Department of Organ Transplant Center, Okayama University Hospital, Okayama, Japan

3 Department of Anesthesiology and Resuscitology, Okayama University Hospital, Okayama, Japan

\section{Introduction}

Lung transplantation (LTx) has been an established treatment for patients with end-stage pulmonary disease for decades [1]. However, accessibility is severely limited by organ availability, and the waitlist mortality remains high. To maximize the survival benefit of LTx in this situation, a recipient selection policy using the lung allocation score (LAS) was implemented in May 2005 by the Organ Procurement and Transplant Network (OPTN) in the United States [2]. Currently, the LAS system is regarded as a generally acceptable allocation policy that can reduce the waiting time of candidates in serious condition and can improve the waitlist mortality in patients with a variety of diagnostic indications for LTx [3]. The concept of the LAS is based mainly on two factors: waitlist urgency and posttransplant survival probability. The policy of urgency-based prioritization clearly contributes to a reduction in the waitlist mortality. Furthermore, some studies that have employed the United States database have concluded that the LAS can predict the posttransplant outcomes [3-6]. 
As with the recipient's condition, the donor status has a considerable influence on the posttransplant outcome due to serious lung injury following cardiopulmonary resuscitation, lung contusion, airway aspiration, and pulmonary infection at the time of brain insult, as well as the presence of underlying lung disease [7]. Therefore, donor factors should be taken into consideration when conducting the validation analysis for the function of the LAS as a predictor of the posttransplant outcome. However, few studies have analyzed the relationship between the LAS and the posttransplant outcomes with the inclusion of detailed donor parameters [8-10]. For donor lung assessment, Oto et al. first proposed a donor scoring system for LTx that was able to successfully predict early posttransplant outcomes [11]. The lung donor score (DS) includes five standard donor criteria factors that are stratified according to severity. This scoring method was validated in previous studies using databases of European and North American LTx centers [12-14].

The United States LAS may be a sophisticated concept that has the potential to provide generalizable insights for different global transplant communities. However, the organ utilization rate varies widely in different countries [15-17], and there is also variability in the donor lung quality among different regions. Therefore, a concept that combines recipient and donor factors should be adopted in validation analyses for the LAS to draw a universal conclusion.

We investigated the function of the LAS as a predictor of the posttransplant outcomes by the donor status, which is represented by the DS.

\section{Materials and methods}

\section{Patients and recipient selection}

This is a retrospective analysis of a consecutive cohort of patients who underwent lung transplantation at Okayama University Hospital, Okayama, Japan, from October 1998 to August 2015. The cohort consisted of 145 patients who received cadaveric lung transplant (CDLTx) or living donor lobar lung transplant (LDLLTx). Patients who had officially approved indications for LTx were basically registered on the waitlist provided by the Japan Organ Transplant Network (JOTN). LDLLTx was considered for critically ill patients who could not await deceased organ donation. All recipients of LDLLTx met the criteria for deceased LTx, and only healthy blood relatives within the third degree or a spouse were accepted as living donors by the institutional review board of Okayama University Hospital. Thirty-seven patients with vascular disease were excluded to eliminate bias related to pretransplant medical management and surgical factors. Clinical data recorded until November 2015 were reviewed following approval of our institutional review board (1605-510).

\section{Donor selection and procurement procedure}

Available cadaveric lungs were allocated to recipients by the Japan Organ Transplant Network according to the waitlist order, ABO compatibility, and matching of the predicted pulmonary function value. Detailed donor data, including the medical history and results of examinations, were obtained by authorized donor coordinators. An experienced transplant physician delegated by the transplant network as a consultant for donor management was involved from the early stage of the allocation process. They collected updated donor information on physical, radiological, and bronchoscopic findings and helped local donor hospital staff optimize the donor condition as much as possible. The final decision on donor selection was made by our experienced transplant physicians. Lung procurement was standardized for all cadaveric and living donors. The lungs were removed en bloc after antegrade perfusion $(60 \mathrm{ml} / \mathrm{kg}$; $4{ }^{\circ} \mathrm{C}, 30 \mathrm{cmH}_{2} \mathrm{O}$ ). Donor lungs were routinely flushed with Modified Eurocollins ${ }^{\circledR}$ (Fresenius, Bad Homburg, Germany) (before 2000) or EP-TU solution ${ }^{\circledR}$ (Cell Science \& Technology Institute, Sendai, Japan) (from 2000) with prostaglandin added to the flush solution. When the lungs were not damaged, additional retrograde perfusion through the pulmonary veins was performed on the back table after returning to the recipient's hospital to optimize lung graft preservation. Similarly, in LDLLTx, antegrade and retrograde perfusion with manual ventilation were performed after procurement of the lower lobe.

\section{Lung transplantation procedure and perioperative management}

Regarding the indications for the procedure, bilateral or single-lung (lobar) transplant was applied for each candidate according to the candidate's primary disease, urgency, and organ availability. Basically, if feasible, single-lung transplant and cadaveric transplant were prioritized over bilateral transplant and living donation from the perspectives of ethicality and the effectiveness of organ utilization. Evidence of pathogenic airway organisms or comorbid pulmonary hypertension was regarded as an indication for bilateral rather than single LTx. Regarding the technical aspects, end-to-end anastomosis with a single running suture was most commonly used. When we performed bilateral LTx, intraoperative cardiopulmonary support with standard bypass technique during pneumonectomy or the implantation of the lung grafts was typically used. Recipients received a triple-drug maintenance immunosuppressive regimen consisting of a calcineurin inhibitor (cyclosporine or tacrolimus), cell-cycle 
inhibitors (azathioprine or mycophenolate mofetil) and steroids. Basiliximab was used as an induction immunosuppressive treatment in recipients with an underlying diminished renal function.

\section{Stratification of the donor lung quality and recipient severity}

The quality of each transplanted lung was retrospectively graded by a scoring method. The DS was defined according to the previous study by Oto et al. [11] (Table 1). In brief, the DS includes five variables: age, smoking history, chest $\mathrm{X}$-ray, secretions, and ratio of arterial oxygen tension to inspired oxygen fraction $\left(\mathrm{PaO}_{2} / \mathrm{FiO}_{2}\right)$. Each variable received a score between 0 and 3 , based on clinical importance, with the exception of $\mathrm{PaO}_{2} / \mathrm{FiO}_{2}$, which was weighted between 0 and 6 . The overall DS score ranged from 0 to 18 . When there were two donors for bilateral LDLLTx, the higher score was adopted. The LAS of each patient was retrospectively calculated in November 2015 to determine recipients' pretransplant severity using the LAS calculator on the OPTN website (https://optn.transplant.hrsa.gov/resou rces/allocation-calculators/las-calculator/). The study population was divided into two groups according to the donor status: the low-DS group, DS $\leq 4$; and the high-DS group, DS $>4$. A DS $>4$ means that at least two variances from the standard donor criteria existed. The two groups were compared with regard to the background clinical variables

Table 1 Donor scoring criteria [11]

\begin{tabular}{lll}
\hline Category & Stratification & Score \\
\hline Age (years) & $<45$ & 0 \\
& $45-54$ & 1 \\
& $55-59$ & 2 \\
& $\geq 60$ & 3 \\
& $<20$ & 0 \\
Smoking history (pack- & $20-39$ & 1 \\
years) & $40-59$ & 2 \\
& $\geq 60$ & 3 \\
Chest X-ray & Clear & 0 \\
& Minor & 1 \\
Secretions & Opacity $\leq 1$ lobe & 2 \\
& Opacity $>1$ lobe & 3 \\
& None & 0 \\
& Minor & 1 \\
$\mathrm{PaO}_{2} / \mathrm{FiO}_{2}$ & Moderate & 2 \\
& Major & 3 \\
& $<450$ & 0 \\
& $351-450$ & 2 \\
& $301-350$ & 4 \\
& $\leq 300$ & 6 \\
\hline
\end{tabular}

(demographics, pulmonary status, surgical variables and donor variables). Correlations between the LAS and posttransplant outcomes (primary graft dysfunction grade, primary $\mathrm{PaO}_{2} / \mathrm{FiO}_{2}$ ratio, length of ventilator support, tracheostomy requirements, length of stay in the intensive care unit, and the survival) were analyzed in each DS group.

\section{Statistical analyses}

Categorical and continuous variables are summarized as the percentage and mean \pm standard deviation. Categorical and continuous variables were compared between donor groups using the chi-squared test or Mann-Whitney $U$ test. Univariate and multivariate regression analyses were performed to determine the influence of various pretransplant clinical variables including the LAS on postoperative outcomes. The survival was calculated via the Kaplan-Meier method and compared with the log-rank test. The conventional $p$ value of 0.05 or less was used to determine the level of statistical significance. All reported $p$ values are two-sided. All analyses were performed with the SPSS software program (SPSS 22.0 for windows: SPSS Inc., Chicago, IL, USA).

\section{Results}

\section{Patient characteristics}

One hundred and eight patients were approved as appropriate candidates for LTx by the institutional review board of Okayama University Hospital. The comparative analysis of patient characteristics with regard to the DS (highDS vs. low-DS) is depicted in Table 2. The mean LAS was $39.1 \pm 7.2$ in the high-DS group and $48.5 \pm 15.3$ in the lowDS group. Patients in the low-DS group were significantly younger and had poorer physical activity than those in the high-DS group and a shorter 6-min walk distance $(<150 \mathrm{ft}$ ). In addition, the time on the waiting list was significantly longer in the high-DS group than in the low-DS group. The leading indication for LTx was idiopathic pulmonary fibrosis (IPF) followed by chronic obstructive pulmonary disease (COPD)/lymphangioleiomyomatosis (LAM), bronchiectasis (BE), and obliterating bronchiolitis (OB) in the high-DS group and IPF followed by OB, COPD/LAM and BE in the low-DS group.

\section{Donor and transplant variables}

The comparative analysis of donor and transplant variables with regard to the DS (high-DS vs. low-DS) is depicted in Table 3. The mean DS was $7.58 \pm 2.4$ in the high-DS group and $1.36 \pm 1.3$ in the low-DS group. The low-DS group included a higher proportion of LDLLTx associated with 
Table 2 Recipient characteristics
Table 3 Donor and transplant variables

\begin{tabular}{llll}
\hline Characteristic & High-DS $(n=34)$ & Low-DS $(\mathrm{n}=74)$ & $p$ value \\
\hline Age of LTx & $42 \pm 13$ & $35 \pm 16$ & 0.03 \\
Age $<15$ & 0 & $13(18 \%)$ & 0.009 \\
Gender, male & $16(47 \%)$ & $30(41 \%)$ & 0.53 \\
Body mass index & $18 \pm 4$ & $17 \pm 5$ & 0.18 \\
Prior thoracic procedure & $16(47 \%)$ & $32(43 \%)$ & 0.71 \\
Chronic steroid use & $12(35 \%)$ & $40(54 \%)$ & 0.07 \\
On ventilator at time of LTx & $11(32 \%)$ & $25(34 \%)$ & 0.88 \\
On ECMO at time of LTx & 0 & $1(2 \%)$ & 0.5 \\
Creatinine level (mg/dL) & $0.7 \pm 0.2$ & $0.5 \pm 0.2$ & 0.002 \\
History of PH & $9(27 \%)$ & $14(19 \%)$ & 0.37 \\
Indication for LTx & & & \\
IPF & $13(38 \%)$ & $34(46 \%)$ & 0.45 \\
COPD & $10(29 \%)$ & $9(12 \%)$ & 0.028 \\
BE & $5(15 \%)$ & $8(11 \%)$ & 0.56 \\
OB & $4(12 \%)$ & $20(27 \%)$ & 0.08 \\
Reoperative LTx & $2(6 \%)$ & $3(4 \%)$ & 0.67 \\
\%VC & $48 \pm 21$ & $44 \pm 24$ & 0.69 \\
Oxygen at rest (L/min) & $2.0 \pm 1.3$ & $2.4 \pm 2.1$ & 0.48 \\
Oxygen at exertion (L/min) & $2.8 \pm 1.3$ & $3.2 \pm 2.4$ & 0.75 \\
6-min walk <150 ft & $2(6 \%)$ & $40(54 \%)$ & $<0.001$ \\
Waiting time (days) & $578 \pm 395$ & $474 \pm 426$ & 0.001 \\
LAS & $39.1 \pm 7.2$ & $4 \pm 15.3$ & \\
\hline
\end{tabular}

$D S$ lung donor score, $L T x$ lung transplant, ECMO extracorporeal membrane oxygenation, $P H$ pulmonary hypertension, $I P F$ idiopathic pulmonary fibrosis, $C O P D$ chronic obstructive pulmonary disease, $B E$ bronchiectasis, $O B$ obliterative bronchiolitis, LAS lung allocation score

\begin{tabular}{lllc}
\hline Variable & High-DS $(n=34)$ & Low-DS $(n=74)$ & $p$ value \\
\hline Donor age (years) & $48 \pm 14$ & $43 \pm 11$ & 0.059 \\
Donor body mass index & $24 \pm 6$ & $24 \pm 4$ & 0.14 \\
Donor smoking history & $20(58 \%)$ & $44(60 \%)$ & 0.95 \\
Donor $\mathrm{PaO}_{2} / \mathrm{FiO}_{2}$ & $345 \pm 105$ & $463 \pm 53$ & $<0.001$ \\
Double $\mathrm{LTx}$ & $24(71 \%)$ & $60(81 \%)$ & 0.33 \\
Single LTx & $10(29 \%)$ & $14(19 \%)$ & $<0.001$ \\
CDLTx & $34(100 \%)$ & $20(27 \%)$ & \\
LDLLTx & $0(0 \%)$ & $54(73 \%)$ & $<0.001$ \\
Maximum ischemic time (min) & $487 \pm 145$ & $248 \pm 164$ & $<0.001$ \\
Ischemic time $>8 \mathrm{~h}$ & $19(56 \%)$ & $10(14 \%)$ & $<0.001$ \\
Ischemic time $>6 \mathrm{~h}$ & $27(79 \%)$ & $16(22 \%)$ & 0.13 \\
CPB use & $26(77 \%)$ & $65(88 \%)$ & 0.13 \\
CPB time (min) & $241 \pm 52$ & $242 \pm 66$ & 0.024 \\
Blood loss (L) & $2.0 \pm 3.0$ & $2.9 \pm 3.3$ & $<0.001$ \\
DS & $7.58 \pm 2.4$ & $1.36 \pm 1.3$ & \\
\hline
\end{tabular}

$\mathrm{PaO}_{2}$ partial pressure of arterial oxygen, $\mathrm{FiO}_{2}$ fraction of inspired oxygen, LTx lung transplant, CDLTx cadaveric lung transplant, $L D L L T x$ living donor lobar lung transplant, $C P B$ cardiopulmonary bypass, DS lung donor score 
a smaller lung volume and shorter organ ischemic time than the high-DS group. Other variables were comparable between the two DS groups.

\section{Correlation between LAS and posttransplant outcomes by DS group}

In the high-DS group, elevated LAS was strongly associated with a poorer $\mathrm{PaO}_{2} / \mathrm{FiO}_{2}$ ratio at $\mathrm{T} 72(p=0.018)$. In the lowDS group, however, there was no association between an elevated LAS and the posttransplant early graft function. A similar trend was observed in the cohort excluding LDLLTx cases (Fig. 1). Univariate analyses examining the correlation between LAS and other early posttransplant outcomes by DS group are shown in Table 4. There was a significant trend in the high-DS group, wherein a high-LAS was associated with a longer duration of ventilator support, ICU stay, oxygen inhalation, and hospital stay after LTx. However, no relationship was found in the low-DS group. A multivariate regression analysis including the LAS and other important clinical variables revealed that the LAS was an independent predictor of early graft performance in the high-DS group but not in the Low-DS group (Table 5).

Regarding the long-term outcome, there was no significant difference in the survival between the two groups (Fig. 2, $p=0.820$ ) with a mean follow-up time of $62 \pm 55$ months (range 3-180 months). During the followup, 23 patients died (high-DS: $n=5 / 34$, low-DS: $n=18 / 74$ ). The survival after 30 days, 1 year, 5 years, and 10 years was $100,89.9,77.6$, and $77.6 \%$ in the high-DS group, respectively, and 98.6, 91.8, 77.8, and 69.6\% in the low-DS group, respectively. Furthermore, when the recipients in the low-DS group were stratified by the LAS (LAS $<50$ or $\geq 50$ ), no significant differences in the survival between the high- and low-LAS groups were observed (Fig. 3). The survival after 1 year, 5 years, and 10 years were $91.8,75.6$, and $64.0 \%$, in the high-LAS patients, respectively, and 91.4, 78.3, and $73.1 \%$, in the low-LAS patients, respectively.

Table 4 Early posttransplant outcomes

\begin{tabular}{|c|c|c|c|c|c|c|}
\hline \multirow[t]{2}{*}{ Variable } & \multicolumn{3}{|l|}{ High-DS $(n=34)$} & \multicolumn{3}{|l|}{ Low-DS $(n=74)$} \\
\hline & High-LAS $(n=5)$ & Low-LAS $(n=29)$ & $p$ value & High-LAS $(n=26)$ & Low-LAS $(n=48)$ & $p$ value \\
\hline Ventilator support (days) & $19 \pm 32$ & $4.0 \pm 10$ & 0.014 & $10 \pm 13$ & $5 \pm 22$ & 0.19 \\
\hline ICU stay (days) & $22 \pm 153$ & $16 \pm 11$ & 0.089 & $21 \pm 18$ & $18 \pm 16$ & 0.40 \\
\hline Oxygen inhalation (days) & $40 \pm 91$ & $24 \pm 16$ & 0.012 & $23 \pm 32$ & $25 \pm 27$ & 0.62 \\
\hline Hospital stay (days) & $118 \pm 74$ & $69 \pm 57$ & 0.054 & $62 \pm 45$ & $79 \pm 45$ & 0.11 \\
\hline 30-day mortality & 0 & 0 & NA & 0 & $1(2 \%)$ & 0.74 \\
\hline 90-day mortality & 0 & $1(3 \%)$ & 0.31 & 0 & $2(4 \%)$ & 0.76 \\
\hline
\end{tabular}

$D S$ lung donor score, $L A S$ lung allocation score, ICU intensive care unit

Table 5 Univariate and multivariate regression analysis for $\mathrm{PaO}_{2} / \mathrm{FiO}_{2}$ ratio at $\mathrm{T} 72$

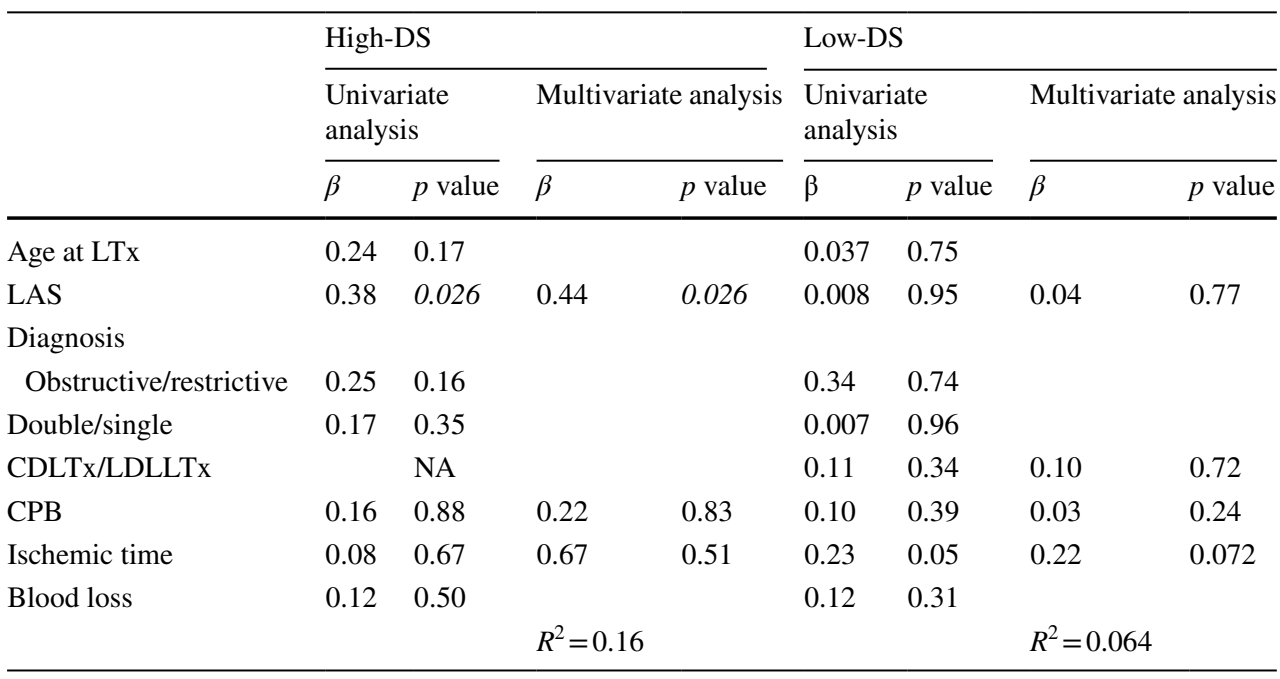

Italic values indicate significance of $p$ value $(p<0.05)$

$\mathrm{PaO}_{2}$ partial pressure of arterial oxygen, $\mathrm{FiO}_{2}$ fraction of inspired oxygen, $\mathrm{DS}$ lung donor score, $\beta$ the coefficient, $L T x$ lung transplant, $L A S$ lung allocation score, CDLTX cadaveric lung transplant, $L D L L T x$ living donor lobar lung transplant, $C P B$ cardiopulmonary bypass, $R^{2}$ the coefficient of determinations 
Fig. 1 Regression analyses between the LAS and posttransplant outcomes. The $\mathrm{PaO}_{2} /$ $\mathrm{FiO}_{2}$ at $72 \mathrm{~h}$ after transplantation (DS lung donor score, $L A S$ lung allocation score, $L T x$ lung transplant, CDLTX cadaveric lung transplant, LDLLTx living donor lobar lung transplant)

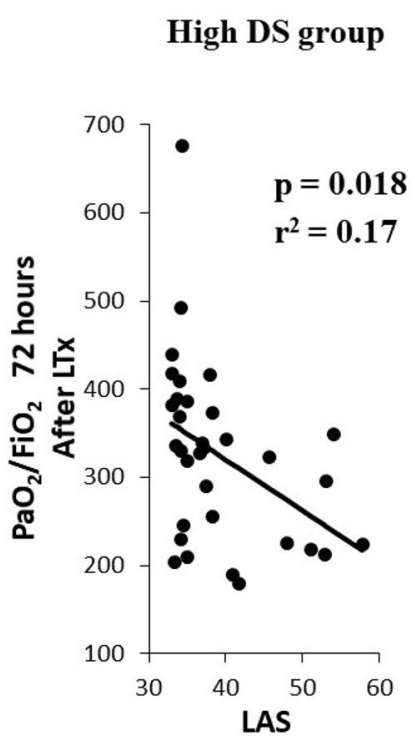

Low DS group

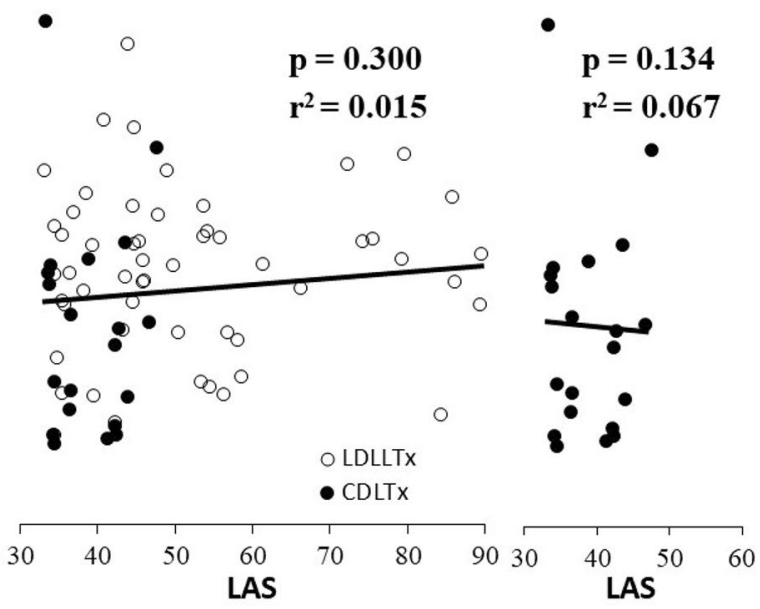

Low DS excluding LDLLTx cases $\mathrm{p}=\mathbf{0 . 1 3 4}$ $=0.067$

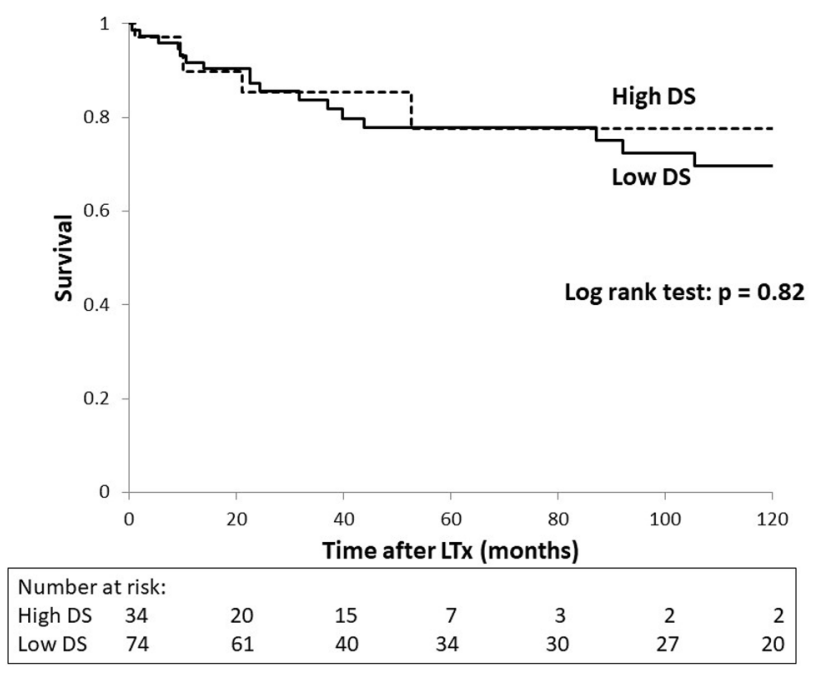

Fig. 2 A Kaplan-Meier analysis of the survival of lung transplant (LTx) recipients stratified by the lung donor score (DS). The number at risk is presented at the bottom of the graph

\section{Discussion}

This study showed that an elevated LAS in the low-DS group was not associated with a worse short-term outcome post-LTx in terms of the lung function and the length of ventilator support; however, an elevated LAS was strongly associated with those parameters in the high-DS group. In the survival analysis for the low-DS group, the high-LAS recipients obtained non-inferiority compared with the lowLAS group. Overall, the LAS system effectively predicted the posttransplant outcome in patients with non-vascular disease only when extended criteria donor lungs with a DS
$>4$ were transplanted. We utilized the DS proposed by Oto et al. [11] and the LAS proposed by the OPTN as benchmarks to grade the condition of lung donors and recipients. We defined patients with a LAS of $\geq 50$ as the high-LAS group based on previous reports that have validated the LAS system [4-6]. A high-DS was set at $>4$, indicating that a donor had multiple variances from the ideal criteria.

The study results are supported by other research suggesting that there is a population in which the LAS is not associated with the post-LTx outcome. Several studies have concluded that patients requiring extracorporeal membrane oxygenation as a bridge to LTx, a markedly high-LAS group, showed comparable survival rates to those who did not have such requirements [18-20]. Furthermore, high-LAS recipients survived significantly longer if two lungs were transplanted than low-LAS recipients who underwent single LTx [21]. These studies also indicate that the ideal condition for lung donation can secure favorable posttransplant outcomes even for high-LAS recipients. Not only the recipient condition but also the total graft performance should be considered when predicting outcomes after LTx.

We adopted a scoring method to objectively stratify the quality of transplanted lungs. In this study, a negative impact of high-DS lungs early after LTx was found, as was described in the original research reported by Oto et al. [11]. While the methodology of scoring the donor status has rarely been applied in past papers, the findings of the present study are reasonable when compared to those of other studies. Sommer et al. reported the importance of selecting stable recipients when marginal lungs are utilized [10]. Mulligan et al. recently reported that the 1-year survival was worse in LTx recipients with an LAS of $\geq 70$ when they received extended criteria donor lungs [8]. Similarly, the results of the current study based on the scoring method for 

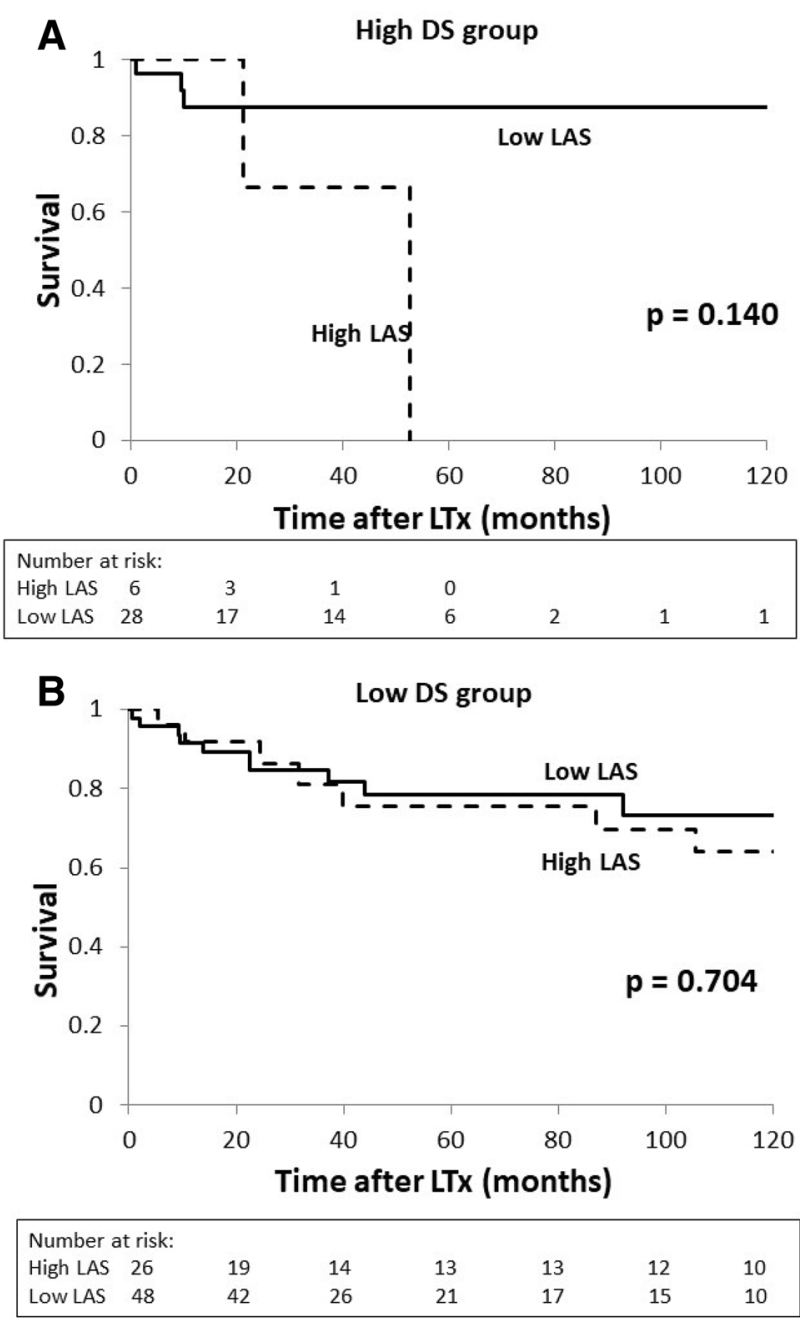

Fig. 3 A Kaplan-Meier analysis of the survival of lung transplant (LTx) recipients stratified by the lung allocation score (LAS) $\mathbf{A}$ in the high-donor score group (high-DS) and $\mathbf{B}$ in the low-donor score group (low-DS). The number at risk is presented at the bottom of the graph

qualifying donor lungs suggest that optimum lung grafts provided acceptable outcomes even in high-LAS recipients and that marginal lungs should not be used in marginal recipients. Reasonable results regarding the correlation between donor/recipient risk-matching and posttransplant outcomes were obtained in this study.

Donor lungs transplanted in our series varied greatly in quality and may, therefore, have represented ideal study subjects. In Japan, since no rescue allocation system or LAS has been established, lung grafts are allocated simply based on the blood type and the order of listing, and $40 \%$ of the patients on the waiting list die without receiving LTx [22]. Historically, the number of cadaveric organ donations in Japan has been extremely low in comparison to other countries $[15,17,23]$. Therefore, peculiar strategies for maximizing the lung utilization rate have been implemented. First, a nationwide lung donor management policy is in operation, sending specialized transplant management doctors to donor hospitals. This system enables lung protection and the acquisition of precise information for donors, leading to a relatively high lung utilization rate ( $68 \%$ per lung) while often using marginal lung grafts (78\% per CDLTx) [16, 24]. The proportion of extended criteria donor lungs for CDLTx in our institution was $81 \%$, which was much higher than that in previous reports $[16,25]$. In addition, $60 \%$ of the marginal lung grafts in our institution met $\geq 2$ extended criteria in terms of the age, smoking history, chest X-ray, secretions, and $\mathrm{PaO}_{2} / \mathrm{FiO}_{2}$. This indicates that the present study includes cases in which severely disqualified lung grafts were transplanted. However, living donors, who generally offer highquality lungs and are classified in the low-DS group, were also included in this study. These unique and wide-ranging donor characteristics in our study provide an ideal study platform for verifying the LAS function and examining the variety of donor/recipient risk-matching models.

Patient selection is key to maintaining healthy posttransplant survival outcomes. Extremely high-LAS patients are likely regarded as unfeasible LTx candidates. However, when focusing on the low-DS $(<4)$ transplant group, we noted no significant difference in the early graft function or the rate of surviving over 10 years between the low- and high-LAS recipients. These data suggest that LAS alone is not an adequate predictor of the posttransplant outcome when quality donor lungs are available. However, at the time of each organ offer, our transplant team has defined transplant candidates' feasibility not only by LAS-related factors but also by their nutritional state, patient frailty, social support, age matching between donor and recipient, and psychological preparation. Although the LAS by itself may be negligible if low-DS lungs are allocated, each decision must be based on other conditions that are not reflected in the LAS mentioned above. Previous studies have suggested that recipient characteristics have a greater impact on the results of LTx than the graft condition [8, 9]. Nevertheless, it is still important that both recipient and donor factors be carefully assessed to identify and optimize the risk of matching between donors and recipients on a case-by-case basis.

Several limitations associated with the present study warrant mention. This was a retrospective study of a singlecenter database in clinical practice over a period of 17 years. We did not account for changes in the lung preservation protocol or recipient management with evolving immunosuppressive regimens over the years. The scale of this study did not allow for a statistical analysis to examine the impact of the LAS on the basis of recipients' primary diseases. However, we removed patients with pulmonary vascular disease from the study, because pretransplant medical management and our operative strategy for patients with pulmonary hypertension changed considerably over the years. In 
addition, we calculated the individual LAS using the LAS calculator provided by the OPTN website/ $\mathrm{UNet}^{\mathrm{SM}}$, as an LAS system has not yet been established in Japan. Finally, the number of LTx recipients included in this study was smaller than in studies from other national databases. Cases with both a high-LAS and high-DS accounted for a small portion of the cohort, which may have affected the results of the regression analyses to a certain extent. A nationwide study with a larger sample size is needed for the further validation of the impact of the donor score on the function of the LAS as a survival predictor after LTx.

In conclusion, LTx can be feasible and provide a survival benefit even for high-LAS patients, provided the lungs from a low-risk donor are transplanted. However, a highLAS with lungs from high-DS donor was associated with a worse primary graft function and a longer ICU and hospital stay than lungs from a low-DS donor. When utilizing low-risk donor lungs, the recipient condition, as evaluated by the LAS system, failed to properly predict the post-LTx outcome.

\section{Compliance with ethical standards}

Conflict of interest None of the authors has a financial relationship with a commercial entity that has an interest in the subject of the presented manuscript or other conflicts of interest to disclose.

\section{References}

1. Arcasoy SM, Kotloff RM. Lung transplantation. N Engl J Med. 1999;340(14):1081-91.

2. Egan TM, Murray S, Bustami RT, Shearon TH, McCullough KP, Edwards LB, et al. Development of the new lung allocation system in the United States. Am J Transplant. 2006;6(5 Pt 2):1212-27.

3. Kozower BD, Meyers BF, Smith MA, De Oliveira NC, Cassivi $\mathrm{SD}$, Guthrie TJ, et al. The impact of the lung allocation score on short-term transplantation outcomes: a multicenter study. J Thorac Cardiovasc Surg. 2008;135(1):166-71.

4. Liu V, Zamora MR, Dhillon GS, Weill D. Increasing lung allocation scores predict worsened survival among lung transplant recipients. Am J Transplant. 2010;10(4):915-20.

5. Russo MJ, Iribarne A, Hong KN, Davies RR, Xydas S, Takayama $\mathrm{H}$, et al. High lung allocation score is associated with increased morbidity and mortality following transplantation. Chest. 2010;137(3):651-7.

6. Horai T, Shigemura N, Gries C, Pilewski J, Bhama JK, Bermudez CA, et al. Lung transplantation for patients with high lung allocation score: single-center experience. Ann Thorac Surg. 2012;93(5):1592-7 (discussion 7).

7. Kotloff RM, Thabut G. Lung transplantation. Am J Respir Crit Care Med. 2011;184(2):159-71.

8. Mulligan MJ, Sanchez PG, Evans CF, Wang Y, Kon ZN, Rajagopal K, et al. The use of extended criteria donors decreases oneyear survival in high-risk lung recipients: a review of the United Network of Organ Sharing Database. J Thorac Cardiovasc Surg. 2016;152(3):891-8.e2.

9. Moreno P, Alvarez A, Santos F, Vaquero JM, Baamonde C, Redel $\mathrm{J}$, et al. Extended recipients but not extended donors are associated with poor outcomes following lung transplantation. Eur J Cardiothorac Surg. 2014;45(6):1040-7.

10. Sommer W, Kuhn C, Tudorache I, Avsar M, Gottlieb J, Boethig D, et al. Extended criteria donor lungs and clinical outcome: results of an alternative allocation algorithm. J Heart Lung Transplant. 2013;32(11):1065-72.

11. Oto T, Levvey BJ, Whitford H, Griffiths AP, Kotsimbos T, Williams TJ, et al. Feasibility and utility of a lung donor score: correlation with early post-transplant outcomes. Ann Thorac Surg. 2007;83(1):257-63.

12. Porro GA, Valenza F, Coppola S, Froio S, Benazzi E, De Fazio N, et al. Use of the Oto lung donor score to analyze the 2010 donor pool of the Nord Italia Transplant program. Transplant Proc. 2012;44(7):1830-4.

13. Smits JM, van der Bij W, Van Raemdonck D, de Vries E, Rahmel A, Laufer G, et al. Defining an extended criteria donor lung: an empirical approach based on the Eurotransplant experience. Transpl Int. 2011;24(4):393-400.

14. Cypel M, Yeung JC, Liu M, Anraku M, Chen F, Karolak W, et al. Normothermic ex vivo lung perfusion in clinical lung transplantation. N Engl J Med. 2011;364(15):1431-40.

15. Israni AK, Zaun D, Bolch C, Rosendale J, Snyder JJ, Kasiske BL. OPTN/SRTR annual data report 2014, deceases organ donation. Am J Transplant. 2016;16:195-215.

16. Hoshikawa Y, Okada Y, Ashikari J, Matsuda Y, Niikawa H, Noda $\mathrm{M}$, et al. Medical consultant system for improving lung transplantation opportunities and outcomes in Japan. Transplant Proc. 2015;47(3):746-50.

17. Eurotransplant International, Foundation. Eurotransplant: donation, waiting lists and transplants. In: Branger P, Samuel U, editors. Annual report 2016 of the Eurotransplant international foundation. 2016. pp 43-66.

18. Hayes D, Tobias JD, Tumin D. Center volume and extracorporeal membrane oxygenation support at lung transplantation in the lung allocation score era. Am J Respir Crit Care Med. 2016;194(3):317-26.

19. Hayanga JW, Lira A, Aboagye JK, Hayanga HK, D'Cunha J. Extracorporeal membrane oxygenation as a bridge to lung transplantation: what lessons might we learn from volume and expertise? Interact Cardiovasc Thorac Surg. 2016;22(4):406-10.

20. Toyoda Y, Bhama JK, Shigemura N, Zaldonis D, Pilewski J, Crespo M, et al. Efficacy of extracorporeal membrane oxygenation as a bridge to lung transplantation. J Thorac Cardiovasc Surg. 2013;145(4):1065-70 (discussion 70-1).

21. Black MC, Trivedi J, Schumer EM, Bousamra M II, van Berkel V. Double lung transplants have significantly improved survival compared with single lung transplants in high lung allocation score patients. Ann Thorac Surg. 2014;98(5):1737-41.

22. Sato M, Okada Y, Oto T, Minami M, Shiraishi T, Nagayasu T, et al. Registry of the Japanese Society of Lung and Heart-Lung Transplantation: official Japanese lung transplantation report, 2014. Gen Thorac Cardiovasc Surg. 2014;62(10):594-601.

23. Soyama A, Eguchi S. The current status and future perspectives of organ donation in Japan: learning from the systems in other countries. Surg Today. 2016;46(4):387-92.

24. Nakagiri T, Inoue M, Minami M, Hoshikawa Y, Chida M, Bando $\mathrm{T}$, et al. Interim report of the Japanese original donor evaluation and management system: the medical consultant system. Surg Today. 2014;44(7):1227-31.

25. Somers J, Ruttens D, Verleden SE, Cox B, Stanzi A, Vandermeulen $\mathrm{E}$, et al. A decade of extended-criteria lung donors in a single center: was it justified? Transpl Int. 2015;28(2):170-9. 